\section{Case Reports in Oncology}

\title{
A Case of Renal Pelvic Cancer with a Complete Duplication of the Renal Pelvis and Ureter
}

\author{
Takuma Nirei Tadashi Tabei Hiroki Ito Kazuki Kobayashi \\ Department of Urology, Yokosuka Kyosai Hospital, Yokosuka, Japan
}

\section{Keywords}

Complete duplication of renal pelvis and ureter $\cdot$ Ectopic ureter $\cdot$ Renal pelvic cancer

\begin{abstract}
This paper describes a case of renal pelvic cancer with a complete duplication of the renal pelvis and ureter, which is substantially rare. A 76-year-old man was referred to the hospital because of gross hematuria for 2 years. A tumor was detected in the upper right kidney using enhanced computed tomography and magnetic resonance imaging scan, and the downstream ureter was suspected to open into the prostate. Retrograde ureteroscopy via the ectopic ureter orifice showed a hemorrhagic papillary tumor consistent with imaging findings. Laparoscopic radical nephroureterectomy was performed and the prostate was preserved because the tumor was only in the renal pelvis. Histopathological examination showed the tumor as a high-grade urothelial carcinoma. There was no sign of recurrence at one and a half years after operation. Ureteroscopy was effective in detecting an upper urinary tract tumor, even via ectopic ureter orifice, and preserving the prostate was possible.
\end{abstract}

(C) 2021 The Author(s).

Published by S. Karger AG, Basel

\section{Introduction}

Duplication of renal pelvis and ureter is the most common urinary tract anomaly. This duplication has two types: complete and incomplete duplication. Occurrence of incomplete duplication is three times as frequent as that of complete duplication, occurring in about one in every 500 people $[1,2]$. While complete duplication has two independent urinary tracts, incomplete duplication has two renal pelvises and ureters which join to form a Y-shaped 
Fig. 1. Ectopic opening to the prostate was suspected by magnetic resonance imaging scan.
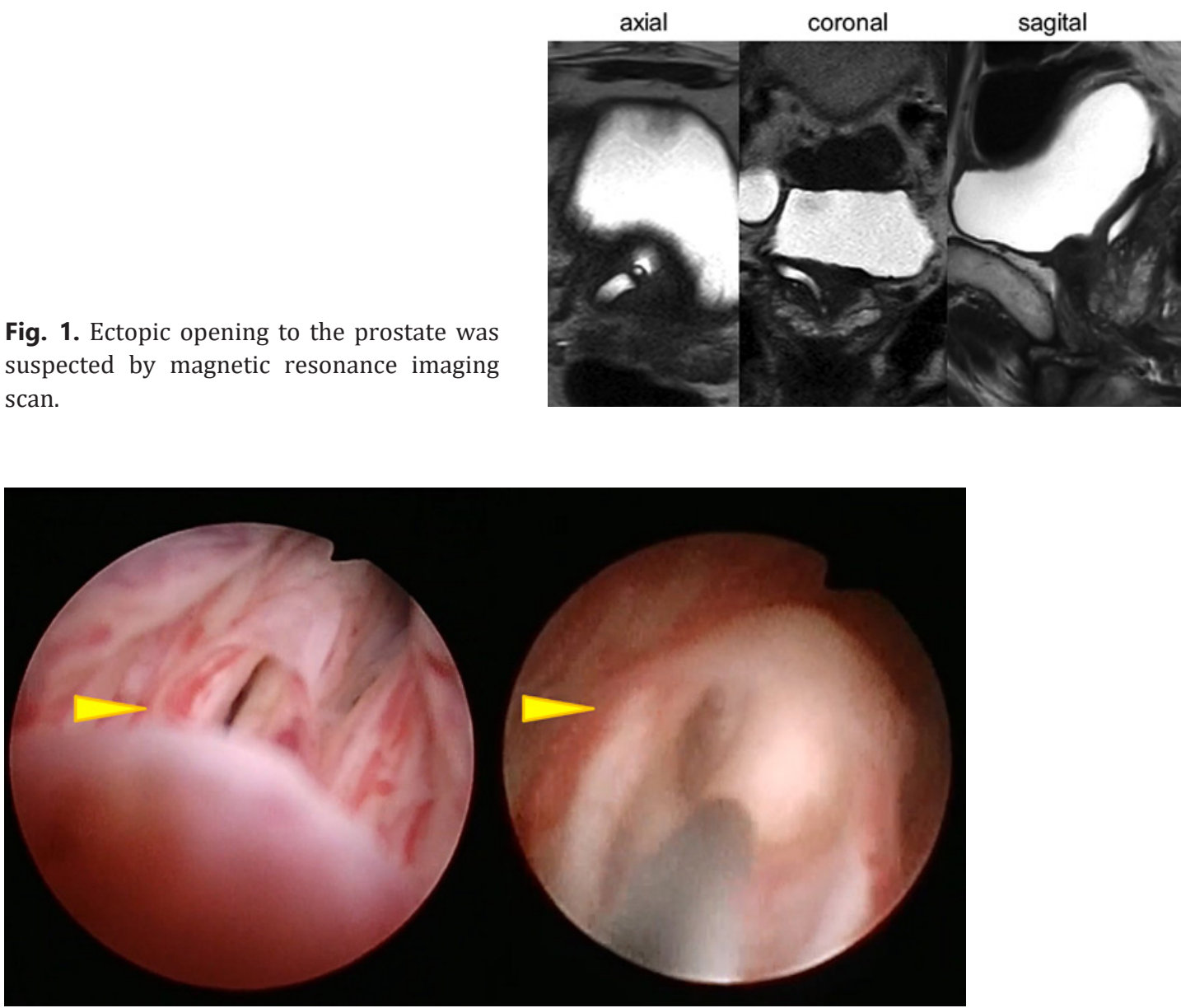

Fig. 2. Ectopic ureter orifice was detected by cystoscope and ureteroscope.

structure. Bladder-ureteral regurgitation is associated with $66-69 \%$ of complete duplications $[3,4]$.

Renal pelvis-ureteral cancer is a relatively rare malignancy, accounting for about $5 \%$ of all urinary tract tumors [5]. It is more prevalent in elderly patients over 50 years of age, and the morbidity rate is twice as high in men than in women [6-8].

Renal pelvis-ureteral carcinoma in patients with complete duplication of the renal pelvis and ureter is substantially rare.

\section{Case Report/Case Presentation}

After suffering gross hematuria for 2 years, a 76-year-old man was referred to the hospital. He had been diagnosed with a complete duplication of the right renal pelvis and ureter in childhood, but had no symptoms. He was taking an antiplatelet drug due to a history of angina. There were no abnormal laboratory findings in blood samples other than a slightly high value of creatinine at $1.04 \mathrm{mg} / \mathrm{dL}$. Abdominal ultrasonography revealed a grade 3 hydronephrosis in the upper right kidney. No tumor was suspected and prostate volume was approximately $34 \mathrm{~mL}$. Flexible cystoscopy did not detect any bladder tumor, renal hematuria, or ectopic orifice. However, an enhanced tumor $15 \mathrm{~mm}$ in diameter in the pelvis of the right 
Fig. 3. A papillary tumor $18 \mathrm{~mm}$ in diameter was detected in renal pelvis of right upper renal unit.

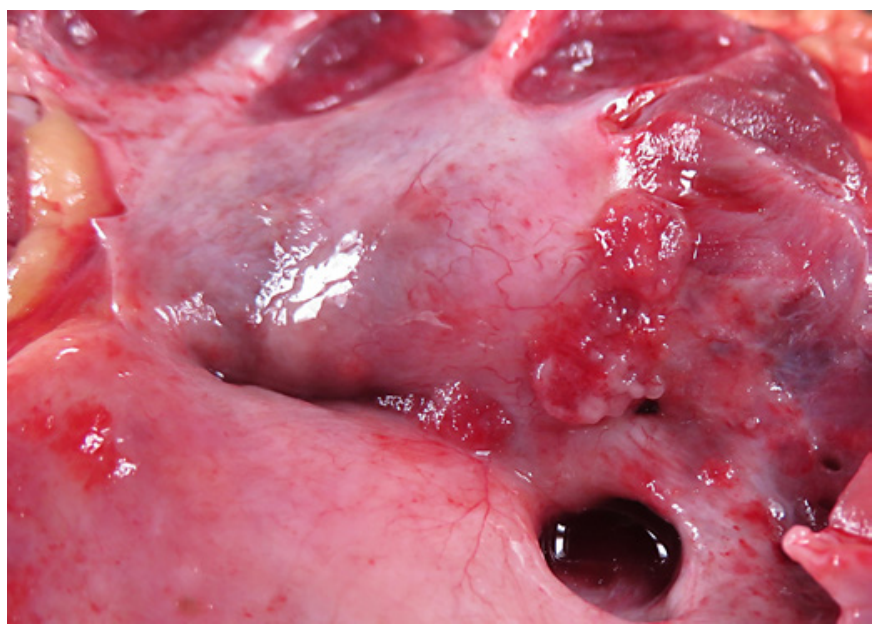

kidney was detected by computed tomography (CT) scan. The ureter from the upper kidney was not open to the bladder and an ectopic opening to the prostate was suspected. This tumor site was mild diffusion reduction in magnetic resonance imaging (MRI) scan. Based on MRI scan shown in Figure 1, it was clearly suspected that the ureter downstream of the tumor was expanding with ectopic opening into the prostate. All urine cytology was negative (class 1 or 2).

Ureteroscopy (URS) under general anesthesia revealed the ectopic ureter opening beside the verumontanum (seminal colliculus), and the findings are shown in Figure 2. A rigid ureteroscope could be inserted smoothly via the ectopic ureter through a hydrophilic guidewire. The lower ureter was expanding but the scope could not be inserted because of meandering. A 10/12-Fr ureter access sheath was able to be inserted without difficulty and the flexible ureteroscope detected a hemorrhagic papillary tumor corresponding to the imaging findings of the renal pelvis. There was no tumor in the ureter. Although renal pelvic urine cytology was negative (class 2), the tumor was suspected to be malignant by findings of URS and imaging, and so laparoscopic radical nephroureterectomy was performed. Because there was no obvious tumor around the ectopic ureter and lower ureter, the lower ureter was blinded and the prostate was preserved. A pedunculated papillary tumor $18 \mathrm{~mm}$ in diameter shown in Figure 3 was detected in the renal pelvis of the right upper renal unit. Histopathological examination of the tumor demonstrated urothelial carcinoma, high grade, pTa. The blind end of the ureter was detected by cystography performed 6 days after surgery, shown in Figure 4.

\section{Discussion/Conclusion}

The complete duplication of renal pelvis and ureter originated from the appearance of two ureteral buds from the Wolff's canal during the embryonic period. The lower hemirenal ureter is often complicated with vesicoureteral reflux and sometimes accompanied by hypoplasia and dysplasia of the kidney in the ureteral opening. On the other hand, there are many cases of ectopic opening and ureteral aneurysms in the upper hemirenal ureter.

In this case, there was a tumor in the renal pelvis in the upper right kidney, which was complete duplication, and downstream ureter was ectopically open to the prostate. It is extremely rare to find renal pelvic cancer with complete duplication of renal pelvis and

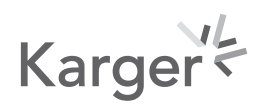




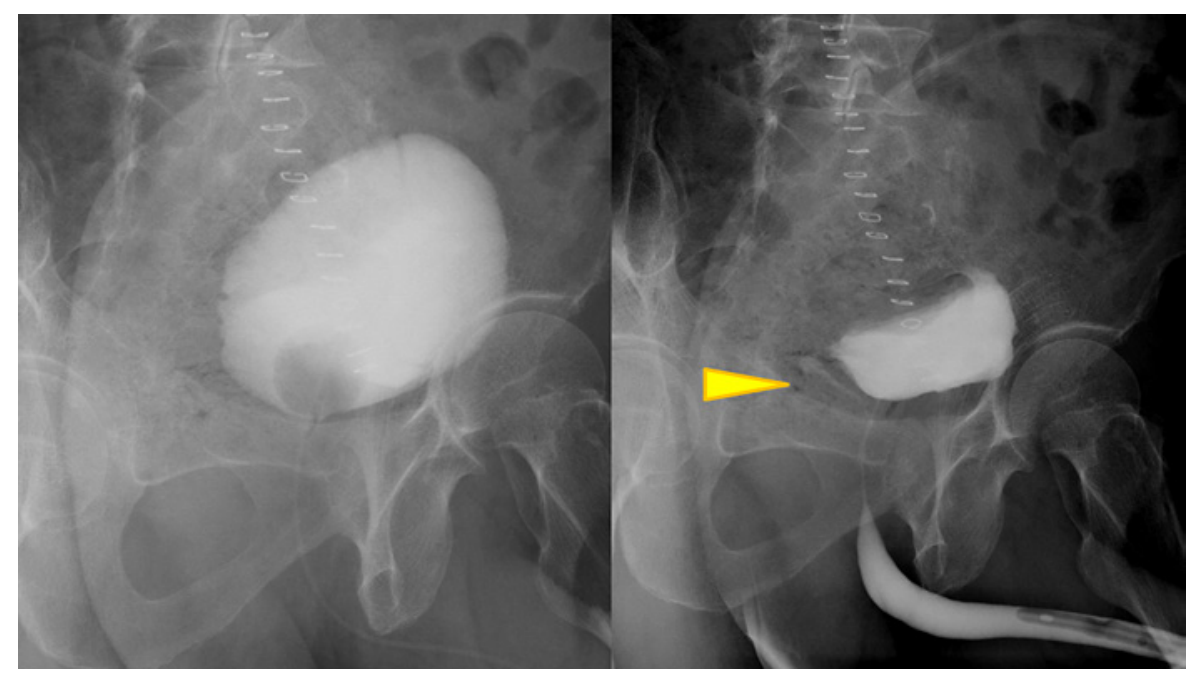

Fig. 4. Blind end of the ureter was detected by cystography.

ureter. In the previous 6 cases of renal pelvic cancer with complete duplication of renal pelvis and ureter, the mean age was $60(44-72)$ years $[9,10]$. Male patients were dominant in those cases ( 5 men/woman) and tumor site were located mostly in left (4/6 tumors) and upper kidneys (6/6 tumors). This case was of a 76-year-old man with tumor in the right side and upper kidney. Of the 3 cases with clear case details, 1 case was diagnosed with imaging findings only, and 2 cases were diagnosed with imaging findings and urine cytology.

Additionally, 5 cases were found of ureteral cancer combining complete duplication of renal pelvis and ureter $[11,12]$. In these total 11 cases, radical nephroureterectomy was performed and there was only one case of ectopic opening to bladder neck. The present case report is the first showing renal pelvis urothelial carcinoma in a patient with complete duplication of renal pelvis and ureter with ectopic opening of ureter to prostate. The renal pelvic tumor was successfully diagnosed by URS in this complicated case. Complications including the unusual site of ectopic ureteral opening and the small tumor which was hardly detected by preoperative imaging. A part of the duplicated ureter on the tumor side was in the prostate and theoretically prostatectomy might be the best treatment option as a simultaneous surgery with nephroureterectomy. However, radical prostatectomy was not performed since the tumor was located only in the pelvis, not ureter. This choice preserved continence and sexual function. There is concern about recurrence of the lower ureter due to the preservation of the lower ureter; thus, close imaging follow-up including CT scan and prostate MRI scan and cystoscopy are being carried out after surgery and there is no recurrence sign at one and a half years after surgery.

Ureteroscopy was effective in detecting an upper urinary tract tumor, even via ectopic ureter orifice, and preserving the prostate was possible.

\section{Acknowledgement}

The authors thank Editage (www.editage.com) for English language editing.

\section{Karger'k}




\section{Case Reports in Oncology}

Case Rep Oncol 2021;14:202-206

\begin{tabular}{l|l}
\hline DOI: $10.1159 / 000513625$ & c 2021 The Author(s). Published by S. Karger AG, Basel
\end{tabular} www.karger.com/cro

Nirei et al.: Renal Pelvic Cancer with Complete Duplication

\section{Statement of Ethics}

Written informed consent was obtained from the patient for the publication of the case report and any accompanying images.

\section{Conflict of Interest Statement}

The authors have no conflicts of interest to declare.

\section{Funding Sources}

This study received no specific grant from any funding agency in the public, commercial, or not-for-profit sectors.

\section{Author Contributions}

All authors were involved in the preparation of this article. All authors have read and approved the final manuscript.

\section{References}

1 Nordmark B. Double formations of the pelves of the kidneys and the ureters: embryology, occurrence and clinical significance. Acta Radiol. 1948;30:276-8.

2 Privett JT, Jeans WD, Roylance J. The incidence and importance of renal duplication. Clin Radiol. 1976;27(4): 521-30.

3 Kaplan WE, Nasrallah P, King LR. Reflux in complete duplication in children. J Urol. 1978;120(2):220-2.

4 Bisset GS 3rd, Strife JL. The duplex collecting system in girls with urinary tract infection: Prevalence and significance. AJR Am J Roentgenol. 1978;148:497-500.

5 Huben RP, Mounzer AM, Murphy GP. Tumor grade and stage as prognostic variables in upper tract urothelial tumors. Cancer. 1988;62(9):2016-20.

6 Anderstom C, Jognsson SL, Pettersson S, Wahlqvist L. Carcinoma of the ureter: a clinicopathologic study of 49 cases. J Urol. 1989;142:280-3.

7 Greenlee RT, Murray T, Bolden S, Wingo PA. Cancer statistics, 2000. CA Cancer J Clin. 2000;50(1):7-33.

8 Hall MC, Sagalowsky AI, Carmody T, Erickstad MD, Roehrborn CG. Prognostic factors, recurrence, and survival in trasitional cell carcinoma of the upper urinary tract: a 30-year experience in 252 patients. Urology. 1998; 52:594-601.

9 Tamada H, Tamura T, Kaneko T, Takata K, Sakuma T, Suzuki Y. [A case of renal pelvic carcinoma in a completely duplicated pelvis and ureter]. Hinyokika Kiyo. 1998;44(10):733-5.

10 Chen KS, Chuang CK, Wu CH, Liaw CC, Lee N. Upper urinary tract tumor in a duplicated collecting system: report of three cases and review of the literature. Chang Gung Med J. 2003 May;26(5):377-82.

11 Takagi S, Gohji K, Iwamoto Y, Masuda H, Segawa N, Kiura H, et al. Ureter cancer of complete double renal pelvis and ureter: a case report. Hinyokika Kiyo. 2002 Dec;48(12):761-4.

12 Unsal A, Cimentepe E, Koc A, Akbulut Z, Balbay MD. A case of primary ureteral carcinoma in association with unilateral complete duplication of the ureter. Int Urol Nephrol. 2003;35(4):489-90.

\section{Karger'}

